\title{
A DOUBLE WINDOW STATE OBSERVER FOR DETECTION AND ISOLATION OF ABRUPT CHANGES IN PARAMETERS
}

\author{
JĘDRZEJ BYRSKI ${ }^{a}$, WITOLD BYRSKI ${ }^{b, *}$ \\ ${ }^{a}$ Department of Applied Computer Science \\ AGH University of Science and Technology, ul. Mickiewicza 30, 30-059 Kraków, Poland \\ e-mail: jbyrski@agh.edu.pl \\ ${ }^{b}$ Department of Automatics and Biomedical Engineering \\ AGH University of Science and Technology, ul. Mickiewicza 30, 30-059 Kraków, Poland \\ e-mail: wby@agh.edu.pl
}

\begin{abstract}
The paper presents a new method for diagnosis of a process fault which takes the form of an abrupt change in some real parameter of a time-continuous linear system. The abrupt fault in the process real parameter is reflected in step changes in many parameters of the input/output model as well as in step changes in canonical state variables of the system. Detection of these state changes will enable localization of the faulty parameter in the system. For detecting state changes, a special type of exact state observer will be used. The canonical state will be represented by the derivatives of the measured output signal. Hence the exact state observer will play the role of virtual sensors for reconstruction of the derivatives of the output signal. For designing the exact state observer, the model parameters before and after the moment of fault occurrence must be known. To this end, a special identification method with modulating functions will be used. A novel concept presented in this paper concerns the structure of the observer. It will take the form of a double moving window observer which consists of two signal processing windows, each of width $T$. These windows are coupled to each other with a common edge. The right-hand side edge of the left-side moving window in the interval $[t-2 T, t-T]$ is connected to the left-hand side edge of the right-side window which operates in the interval $[t-T, t]$. The double observer uses different measurements of input/output signals in both the windows, and for each current time $t$ simultaneously reconstructs two values of the statethe final value of the state in the left-side window $z_{T}(t-T)$ and the initial value of the state $z_{0}(t-T)$ in the right-side window. If the process parameters are constant, the values of both the states on the common joint edge are the same. If an abrupt change (fault) in some parameter at the moment $t_{A}=t-T$ occurs in the system, then step changes in some variables of the canonical state vector will also occur and the difference between the states will be detected. This will enable localization of the faulty parameter in the system.
\end{abstract}

Keywords: fault detection and isolation, exact state observer, parameter abrupt changes, derivative reconstruction, linear continuous systems.

\section{Introduction}

Scientists are inventing more and more complicated algorithms for diagnosis and detection of faults in industrial processes. The literature on this subject is very rich (e.g., Frank, 1990; Chen and Patton, 1999; Patton et al., 2000; Chiang et al., 2001; Blanke et al., 2003; Korbicz et al., 2004; Isermann, 2006; Ding, 2013). On-line application of these algorithms requires modern computers and advanced information processing systems.

\footnotetext{
${ }^{*}$ Corresponding author
}

One of the problems of interest is detection and isolation of abrupt changes in some intrinsic and real parameters of technological objects, which may be of importance for both safety and economic reasons.

Usually, two types of faults may occur-measuring instruments or actuator faults and process faults. For simulation purposes, instrument faults can be modeled as additive faults, whereas process faults are mainly multiplicative ones. If instrument faults are dangerous for some safety-critical operation, a commonly used approach is the use of extra redundant measurement 
hardware such as extra sensors, transducers, measuring buses and indicators, and even triple-modular redundancy (TMR) technology. Then the concept of voting is used (two-out-of-three, 2oo3) for guaranteeing safety integrity level SIL3 or SIL4 according to the norm EC61508/61511. Other important conditions for safety of programmable control systems can be found in the norm PN-EN 62061.

If, however, there is no possibility of using extra hardware redundant equipment and simultaneously the working sensors are not reliable and of high quality, then one can implement the idea of additional virtual soft-sensors for instrumentation fault diagnosis and isolation (FDI). Sometimes virtual soft-sensors can be very sophisticated and need special methods of signal processing. For example, reconstruction of the derivative from the measured signal is a serious problem, especially if this signal contains noise and other disturbances. Consequently, if a parametric model of the dynamic system is known and all the state variables as well as the input and output signals are accessible for measurements, and also good quality of all the sensors (hardware or virtual) is assured, then recognition of process faults and emergency situations is usually not that difficult (Wang et al., 2008).

However, if all of the process variables are not measured, then the procedures for process fault diagnosis and isolation may be not easy.

Summarizing the above, one can present two different schemes of process faults diagnosis to represent two different situations.

Case 1. All the process variables are measured (such as state variables and input/output signals).

(a) Assume that there is also the possibility of supplying the whole process with a full set of highly reliable hardware sensors and instrumentation (even hardware-redundant sensors) for correct, accurate and error-free measurements. In such a case, detection of some differences (residuals) between the nominal relationship of certain process values arising from the first principles and simple reasoning rules enables confident process fault diagnosis and isolation by the FDI system.

(b) Assume that the whole process is equipped only with a full set of single measurement sensors for all the process variables. These sensors, however, do not guarantee error-free measurements and hardware redundancy is not available, so one can apply some additional virtual soft-sensors (the use of analytical redundancy) which enable a comparison of measurement estimates with real measurements and a confirmation of the error-free sensor operation. In such a case, detection of process faults by the FDI system has a similar difficulty level as in Case 1(a).
Case 2. Not all the process variables are measured. For instance, very often the internal state $x$ is unmeasurable and only the input $u$ and the output $y$ are measured. In such a case, the detection of some process faults is not easy, although not impossible. Let us at least assume that high quality instrumentation is used and the correct and reliable input/output measurements are available. Then the FDI system usually uses a model-based approach with implementation of different methods for modeling, parameter identification, state observation and different hypotheses testing.

This paper will focus on diagnosis of faults in continuous processes as in Case 2 above.

Generally, for such cases, many different methods based on dynamic system modelling have been investigated and various state observation and identification algorithms used (e.g., Chen and Zhang, 1991; Ding and Guo, 1996; Simani et al., 2003; Rolink et al., 2006; Ding, 2013).

Here it will be assumed that in the continuous system abrupt changes in parameters will occur. The values of the parameters before and after the change are constant. The changes are not catastrophic, but take the form of a sudden modification of the parameters' values, which changes the dynamics of the system. The detection of such very fast changes is very important and was investigated in the literature some years ago (Basseville and Nikiforov, 1993; Niedzwiecki, 1994; Lai and Shan, 1999). However, the importance of this problem still remains valid and the results of the research are still being published, both for discrete systems (Costa et al., 2005; Ukil and Zivanovic, 2007) as well as for continuous ones (Costa et al., 2013).

As mentioned previously, a new method of fault detection will be presented in this paper, and it will be applied to linear continuous systems. Assume that in this system abrupt changes in parameters occur at the moment $t_{A}$. It will be proven that such parameter changes will result in step changes of some derivatives $y^{(i)}\left(t_{A}\right)$ of the output signal $y(t)$. Careful observation of these derivatives and recognition of their order in which step changes occur will provide information about the location of the faulty parameter in the structure of the system.

\section{Reconstruction of derivatives}

One of the main elaborated research issues in this paper will be a new method of reconstructing function derivatives. Generally, numerical differentiation plays an important role in data processing; however, the design of an ideal differentiator working on noisy data is a difficult problem and is often known to be ill-posed (small noise can induce a big error in the approximated derivatives).

Various methods for determination of the derivative from measurement signals have been proposed in various research publications. 
In some works (e.g., Carlsson et al., 1991), a stochastic approach to the problem is used. In others (Qu, 1996), an algebraic approximation method for differentiation is proposed. In the paper of Vainio et al. (1997), one can find the use of finite impulse response (FIR) filters as well as least-squares linear and quadratic approximation to obtain output signal derivatives. For extracting the signs of derivatives, bond-graphs were used. Smith et al. (2003) elaborated a method of algebraic computation of derivatives in frequency domain. Ibir (2004) proposed use of a high-gain asymptotic observer for derivative reconstruction, and in the work of Wei et al. (2005) scattered noisy data were taken into consideration.

Reger and Jouffroy (2009) studied the method of using the Gram observability matrix for calculation of derivatives from the function $y(t)$ and use of an integral operator. In this work, the function $y(t)$ is interpreted as the output of an autonomous system with no control signal with multiple zero eigenvalues in the state matrix. The presented method enables reconstruction of the output initial conditions $y(0)$. Byrski and Fuksa (1996) presented for the first time the problem of simultaneous identification of system parameters and state observation by use of a modulating function. A similar problem was investigated by Jouffroy and Reger (2015).

In this paper, we will use the theory of exact state observers for on-line reconstruction of the derivatives $y^{(i)}(t)$ of the signal $y(t)$. This signal will be the output signal of any continuous linear SISO system driven by the control signal $u(t)$. For reconstruction of these derivatives, a special type of integral state observer will be used. These observers can reconstruct the exact value of the state, and so differ from differential estimators such as the Luenberger observer or the Kalman filter. Such estimators introduce additional uncertainty to the reconstructed state because of their asymptotic nature (the estimate of the state error converges to zero asymptotically). Thus, use of such estimators for derivative estimation as well as diagnosis of the system may be ineffective.

Fast modern computers make application of other on-line observation algorithms possible. They originate directly from the definition of state observability. The theory of the general and optimal form of the exact state observer with a minimal norm, formulated in Hilbert spaces, was presented for the first time by Fuksa and Byrski (1984). Other versions and different applications were presented later on by Byrski $(1995 ; 2003)$. The most general structure of such an observer is given by the inner product (the integral) of the measurements of the output $y \in Y$ and input $u \in U$ as well as special observation matrix functions $G_{1}(\tau), G_{2}(\tau)$ on interval $[0, T]$. After the first observation interval $[0, T]$, the observer reconstructs the exact value of the finite state $x(T)$. Using the moving window observer MWO on interval $[t-T, t]$, it is possible to observe $x(t)$ on-line for every $t>T$. After the first observation interval $[0, T]$, one can also reconstruct the initial state $x(0)$ with the use of other functions $\bar{G}_{1}(t), \bar{G}_{2}(t)$ and the same input/output signals. There are many different special observation pairs of functions $G_{1}(t), G_{2}(t)$ or $\bar{G}_{1}(t), \bar{G}_{2}(t)$ which fulfill the definition of the exact observer that exactly reconstructs the state. In the above-mentioned publications, the use of an observer which has a minimal norm in the space $L^{2}[0, T]$ was recommended. Such an observer ensures the occurrence of the smallest state reconstruction error in the case of disturbances in measurements of $y$ and $u$.

In this paper, the exact state observer for reconstruction of the canonical state vector $z(t)$ is used. The elements of this state are the derivatives $y^{(i)}(t)$ of the output signal $y(t)$. Detection of step changes in the state variables will enable detection of rapid changes in the system parameters.

\section{Use of the double window observer}

Assume that, in the system, abrupt changes in parameters occur at a moment $t_{A}$. It will result in step changes of the derivatives of output signal $y^{(i)}\left(t_{A}\right)$. Exact state observation and recognition of the order of the derivative in which the step changes occur will provide information about the location of the faulty real parameter in the system structure. To this end, the new concept of a moving double window state observer is used. This observer is based on two windows coupled to each other with a common middle edge. The right-hand side edge of the left window, which is defined in interval $[t-$ $2 T, t-T]$, follows the left-hand side edge of the right window, which operates in interval $[t-T, t]$. The observer works simultaneously within both the windows with different measurements of input/output signals given on the intervals $[t-2 T, t-T]$ and $[t-T, t]$, respectively ( $t$ denotes the current time). The left window observer uses the special observation matrix functions $G_{1}(\tau)$, $G_{2}(\tau)$ (for reconstruction of the final state $z_{T}$ ), and the right window observer uses the functions $\bar{G}_{1}(\tau), \bar{G}_{2}(\tau)$ (for reconstruction of the initial state $z_{0}$ ).

If the system parameters $a_{i}, b_{i}$ are constant, the values of both the reconstructed states are the same, $z_{T}(t-T)=z_{0}(t-T)$. If abrupt changes (faults) of parameters in the system occur at the moment $\left(t_{A}=\right.$ $t-T)$ then step changes in the state occur and the double observer will detect the difference between the states $z_{T}\left(t_{A}\right) \neq z_{0}\left(t_{A}\right)$. This will be possible, because before the moment of fault occurrence $t<t_{A}$ the model parameters are known (identified); hence, the observation matrices $G_{1}(\tau), G_{2}(\tau)$ are also known. For $t>t_{A}$ immediately after detection of time $t_{A}$ by the FDI system, the procedure for identification of the new value of model parameters within the identification window 
$\left[t_{A}, t_{A}+T_{I D}\right]$ will start. The fast identification method for continuous systems was published in our previous works (Byrski and Byrski, 2012b). It is based on a modulating function procedure and works within an extra processing window of width $T_{I D}<T$. After the identification, the new value of model parameters $\bar{a}_{i}, \bar{b}_{i}$ will be used for calculation of the new observation matrices $\bar{G}_{1}(t), \bar{G}_{2}(t)$ and reconstruction of $z_{0}\left(t_{A}\right)$, enabling proper detection of the difference $z_{T}\left(t_{A}\right) \neq z_{0}\left(t_{A}\right)$. This detection will be possible at the current moment of time $t=t_{A}+T$, which means some short delay $T$ after the moment of parameter fault occurrence $t_{A}$.

To complete this section, one more important remark needs to be made. In this paper, we will discuss two different classes of parameters. Abrupt changes (faults) affect real parameters such as electrical resistance, capacity or flow rate coefficients $S_{i}$ in hydraulic systems. On the other hand, the identification procedure identifies the transfer function of the system, which means the parameters $a_{i}, b_{i}$ of the input/output model equation:

$$
\begin{aligned}
y^{(n)}(t)+a_{n-1} & y^{(n-1)}(t) \\
& +\cdots+a_{1} y^{(1)}(t)+a_{0} y(t)=b_{0} u(t) .
\end{aligned}
$$

Such model parameters $a_{i}, b_{i}$ can be called 'aggregated parameters' because they are nonlinear functions of the real parameters $S_{i}$. Very often isolation of the faulty parameter $S_{i}$ by solving the set of nonlinear equations is impossible because of the ambiguity of calculation.

One of the important contributions of this paper is to show that the proposed method indicates, in the structure of the model, the real parameter $S_{i}$ which fails, and for this purpose it only needs the new identified values of the model aggregated parameters $\bar{a}_{i}, \bar{b}_{i}$ and does not require knowledge of the new value of parameter $S_{i}$. The number $i$ of the real parameter $S_{i}$ which has failed is indicated by the step in the $i$-th derivative.

\section{Class of systems under consideration}

The linear time invariant (LTI) model of the continuous, observable and controllable single-input-single-output (SISO) system with the real state $x$ and output $y$ is given by the formulas

$$
\begin{aligned}
\dot{x}(t) & =A x(t)+B u(t), \quad x\left(t_{0}\right)=x_{0}, \\
y(t) & =C x(t),
\end{aligned}
$$

where $x(t) \in \mathbb{R}^{n}, y(t) \in \mathbb{R}, u(t) \in \mathbb{R}, \forall t \geq 0$.

After linear similarity transformation of the real state $x$ by the observability matrix $Q_{O}$, one can obtain the new canonical vector state $z(t): z(t)=Q_{O} x(t)$, where

$$
\begin{gathered}
Q_{O}=\left[\begin{array}{c}
C \\
C A \\
\vdots \\
C A^{n-1}
\end{array}\right], \\
F=Q_{O} A Q_{O}^{-1}=\left[\begin{array}{cccc}
0 & 1 & 0 & 0 \\
0 & 0 & 1 & \cdots \\
\vdots & \vdots & \vdots & 1 \\
-a_{0} & -a_{1} & \cdots & -a_{n-1}
\end{array}\right] .
\end{gathered}
$$

The new canonical system with the same input $u(t)$ and output $y(t)$ as in (1) has the Frobenius state matrix $F$ and matrices $B_{F}, C_{F}$ and is also state observable:

$$
\begin{aligned}
& \dot{z}(t)=F z(t)+B_{F} u(t), \quad z\left(t_{0}\right)=Q_{O} x_{0}, \\
& y(t)=C_{F} z(t),
\end{aligned}
$$

$$
\begin{gathered}
B_{F}=Q_{O} B=\left[\begin{array}{c}
0 \\
0 \\
\vdots \\
b_{0}
\end{array}\right], \\
C_{F}=C Q_{O}^{-1}=\left[\begin{array}{lllll}
1 & 0 & 0 & \ldots & 0
\end{array}\right]
\end{gathered}
$$

The corresponding transfer function is

$$
\begin{aligned}
G(s) & =C[s I-A]^{-1} B=C_{F}[s I-F]^{-1} B_{F} \\
& =\frac{b_{0}}{s^{n}+a_{n-1} s^{n-1}+\cdots+a_{1} s+a_{0}},
\end{aligned}
$$

and the $n$-th order differential equation for the output $y(t)$ is

$$
\begin{aligned}
y^{(n)}(t)+a_{n-1} & y^{(n-1)}(t) \\
& +\cdots+a_{1} y^{(1)}(t)+a_{0} y(t)=b_{0} u(t) .
\end{aligned}
$$

For the state $z(t)$, the following well-known equality is fulfilled:

$$
z(t)=\left[\begin{array}{c}
z_{1}(t) \\
z_{2}(t) \\
\vdots \\
z_{n}(t)
\end{array}\right]=\left[\begin{array}{c}
z_{1}(t) \\
\dot{z}_{1}(t) \\
\vdots \\
z_{1}^{(n-1)}(t)
\end{array}\right]=\left[\begin{array}{c}
y(t) \\
\dot{y}(t) \\
\vdots \\
y^{(n-1)}(t)
\end{array}\right] .
$$

The state variables $z_{i}(t)$ represent consecutive derivatives of the output $y(t)$.

\section{Theory of exact integral observers}

Based on the work of Byrski (2003), the main formulas for integral state observers will be recalled. Assume that we measure the control $u$ and the output $y$ of the system on the interval $[0, T]$, where $T$ is the fixed observation time 
(regardless of whether it is a system working in open loop or closed loop). Our purpose is to determine the initial state $z(0)$ which will be reconstructed at the beginning of the operating window. The output $y(t)$ of the real system (1) may be also calculated as the output of the canonical system (2) for $t \in[0, T]$ based on the well-known formula with the initial condition $z(0)$ and control signal $u(t)$ :

$$
y(t)=C_{F} e^{F t} z(0)+C_{F} \int_{0}^{t} e^{F(t-\tau)} B_{F} u(\tau) \mathrm{d} \tau
$$

We shall derive one of possible formulas of integral observer for reconstruction of the exact value of the initial state $z(0)$. To this end, one should multiply both the sides of Eqn. (4) by the transposed matrix $\left[C_{F} e^{F t}\right]^{\prime}$, and next integrate such an equation over the interval $[0, T]$. Because of the assumption of state observability, the real Gram matrix $M_{F 0}$ is nonsingular for any $T$ :

$$
M_{F 0}=\int_{0}^{T} e^{F^{\prime} t} C_{F}^{\prime} C_{F} e^{F t} \mathrm{~d} t .
$$

Hence, the initial state $z(0)=z_{0}$ may be reconstructed exactly by the formula

$$
z(0)=\int_{0}^{T} \bar{G}_{F 01}(\tau) y(\tau) d \tau+\int_{0}^{T} \bar{G}_{F 02}(\tau) u(\tau) \mathrm{d} \tau .
$$

Equation (5) as well as the matrices $\bar{G}_{F 01}(\tau)$ and $\bar{G}_{F 02}(\tau)$ (vectors in the SISO case) result from the above transformation of (4). During this operation, in the second part of (4) one should change the integration order. The formula (5) represents the exact integral observer, which is given by two integrals with products of function vector $\bar{G}_{F 01}(\tau)$ and the output signal as well as $\bar{G}_{F 02}(\tau)$ and the input signal. These vectors $\bar{G}_{F 01}(\tau)$ and $\bar{G}_{F 01}(\tau)$ are defined as in

$$
\begin{aligned}
& z(0) \\
& =\int_{0}^{T} M_{F 0}^{-1} e^{F^{\prime} \tau} C_{F}^{\prime} y(\tau) \mathrm{d} \tau \\
& -\int_{0}^{T}\left[\int_{\tau}^{T} M_{F 0}^{-1} e^{F^{\prime} s} C_{F}^{\prime} C_{F} e^{F(s-\tau)} \mathrm{d} s\right] B_{F} u(\tau) \mathrm{d} \tau .
\end{aligned}
$$

For the known (identified) system matrices $F, B_{F}, C_{F}$, the observer vectors $\bar{G}_{F 01}(\tau)$ and $\bar{G}_{F 02}(\tau)$ (matrices in the multidimensional case) may be calculated off-line in interval $[0, T]$. Then, for the given output $y(t)$ and input $u(t)$ in $[0, T]$, it is easy to find the initial state $z(0)$ from
(5). Such an initial state in $[0, T]$ will be reconstructed by the right-side window of the double observer.

The same calculation procedure may be used for derivation of the exact value of the final state $z(T)$ in the window $[0, T]$. The formula for the output $y(t)$, which is based on the unknown final condition $z(T)$, is analogous to Eqn. (4) and is of the form

$$
\begin{aligned}
y(t)= & C_{F} e^{-F(T-t)} z(T) \\
& -C_{F} \int_{t}^{T} e^{F(t-\tau)} B_{F} u(\tau) \mathrm{d} \tau .
\end{aligned}
$$

In a similar manner, after some calculation one can obtain the form of the integral observer for exact reconstruction of the final state $z(T)=z_{T}$ of the window:

$$
z(T)=\int_{0}^{T} G_{F T 1}(\tau) y(\tau) \mathrm{d} \tau+\int_{0}^{T} G_{F T 2}(\tau) u(\tau) \mathrm{d} \tau,
$$

where the observer matrices are

$$
\begin{aligned}
& G_{F T 1}(\tau)=e^{F T} M_{F 0}^{-1} e^{F^{\prime} \tau} C_{F}^{\prime}, \\
& G_{F T 2}(\tau)=e^{F T} M_{F 0}^{-1}\left[\int_{0}^{\tau} e^{F^{\prime} s} C_{F}^{\prime} C_{F} e^{F s} d s\right] e^{-F \tau} B_{F} .
\end{aligned}
$$

Such a value of the final state of the window will be reconstructed by the left window of the double observer. Based on the above formulas, one can derive an on-line version of the double window state observer, which operates for the current $t$, in the interval $t \in\left[T, T_{F}\right]$ according to the following formulas:

- in the left window for reconstruction of the final state,

$$
\begin{aligned}
z_{T}(t-T)= & \int_{0}^{T} G_{F T 1}(\tau) y(t-2 T+\tau) \mathrm{d} \tau \\
& +\int_{0}^{T} G_{F T 2}(\tau) u(t-2 T+\tau) \mathrm{d} \tau,
\end{aligned}
$$

- in the right window for reconstruction of the initial state,

$$
\begin{aligned}
z_{0}(t-T)= & \int_{0}^{T} \bar{G}_{F 01}(\tau) y(t-T+\tau) \mathrm{d} \tau \\
& +\int_{0}^{T} \bar{G}_{F 02}(\tau) u(t-T+\tau) \mathrm{d} \tau .
\end{aligned}
$$


If the system parameters are constant and known, the reconstructed value of the final state in the left-side window and the initial state in the right-side window are the same: $z_{T}(t-T)=z_{0}(t-T)$, see Fig. 1. In order not to obscure the figure, only the plot of the output signal $y$ was drawn. The plot of the control signal $u$ was omitted.

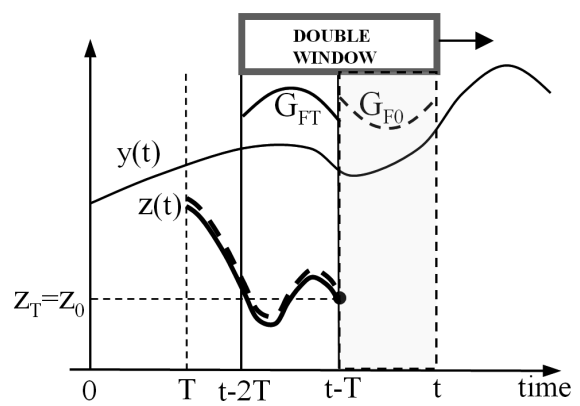

Fig. 1. Double window state observer (no faults).

If, in the observed system, the abrupt changes (faults) of its parameters at the moment $t_{A}$ occur, then the step changes in the state will also occur, and after time $T$ $\left(t=t_{A}+T\right)$ the double window observer will detect the difference of the border states $z_{T}(t-T) \neq z_{0}(t-T)$ (between the windows) on its common edge, see Fig. 2. In this figure the entire plots of the two reconstructed states $z_{T}$ and $z_{0}$ are presented, which are the result of the on-line action of the observer in the entire interval $\left[T, T_{F}\right]$. The position of the observer was plotted for $t=t_{A}+T$.

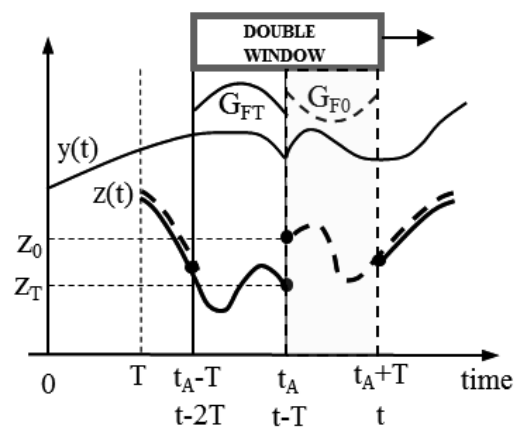

Fig. 2. Double window state observer (parameter fault).

It should be pointed that after detection of the parameter fault at $t=t_{A}$, in the moving double window observer, the right-side window must be switched off from on-line operation in open interval $t \in\left(t_{A}, t_{A}+T\right)$ (Fig. 2, the dashed line), because of outdated parameter data, and the left-side window must be switched off for $t \in\left(t_{A}+T, t_{A}+2 T\right)$ (the solid line). Such a rule of observer operation guarantees state reconstruction to be executed by the double window observer constantly and without any breaks.

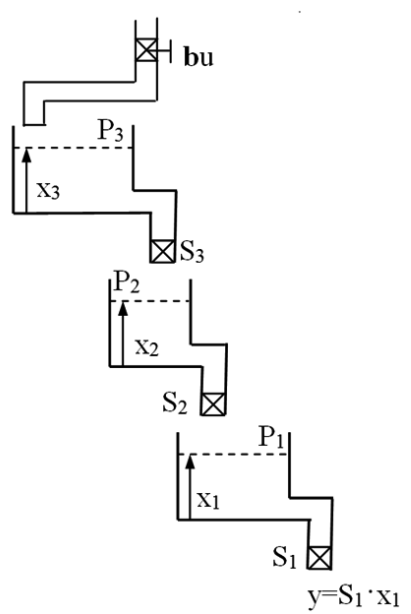

Fig. 3. Three tank cascade system.

\section{First example system with faults}

As the first example of (1) we shall consider a three tank cascade connected system as shown in Fig. 3, given by

$$
\begin{gathered}
{\left[\begin{array}{l}
\dot{x_{1}}(t) \\
\dot{x_{2}}(t) \\
\dot{x_{3}}(t)
\end{array}\right]=\left[\begin{array}{ccc}
-\frac{S_{1}}{P_{1}} & \frac{S_{2}}{P_{1}} & 0 \\
0 & -\frac{S_{2}}{P_{2}} & \frac{S_{3}}{P_{2}} \\
0 & 0 & -\frac{S_{3}}{P_{3}}
\end{array}\right]} \\
\times\left[\begin{array}{l}
x_{1}(t) \\
x_{2}(t) \\
x_{3}(t)
\end{array}\right]+\left[\begin{array}{c}
0 \\
0 \\
\mathbf{b} / P_{3}
\end{array}\right] u(t), \\
y(t)=\left[\begin{array}{ll}
S_{1} & 0
\end{array}\right] x(t), \\
G(s)=\frac{b_{0}}{s^{3}+a_{2} s^{2}+a_{1} s+a_{0}} \\
=\frac{b_{0}}{\left(s+\frac{S_{1}}{P_{1}}\right)\left(s+\frac{S_{2}}{P_{2}}\right)\left(s+\frac{S_{3}}{P_{3}}\right)},
\end{gathered}
$$

$$
\begin{aligned}
b_{0} & =\mathbf{b} \frac{S_{1} S_{2} S_{3}}{P_{1} P_{2} P_{3}}, \\
a_{2} & =\frac{S_{1}}{P_{1}}+\frac{S_{2}}{P_{2}}+\frac{S_{3}}{P_{3}}, \\
a_{1} & =\frac{S_{1} S_{2}}{P_{1} P_{2}}+\frac{S_{1} S_{3}}{P_{1} P_{3}}+\frac{S_{2} S_{3}}{P_{2} P_{3}}, \\
a_{0} & =\frac{S_{1} S_{2} S_{3}}{P_{1} P_{2} P_{3}} .
\end{aligned}
$$

The observability matrix $Q_{O}$ and matrices $F, B_{F}$, 
$C_{F}$ are

$$
\begin{aligned}
& Q_{O}=\left[\begin{array}{c}
C \\
C A \\
C A^{2}
\end{array}\right] \\
& =S_{1}\left[\begin{array}{ccc}
1 & 0 & 0 \\
\frac{-S_{1}}{P_{1}} & \frac{S_{2}}{P_{1}} & 0 \\
\frac{S_{1}^{2}}{P_{1}^{2}} & \frac{-S_{1} S_{2}}{P_{1}^{2}}-\frac{S_{2}^{2}}{P_{1} P_{2}} & \frac{S_{2} S_{3}}{P_{1} P_{2}}
\end{array}\right] \text {, } \\
& F=Q_{O} A Q_{O}^{-1}=\left[\begin{array}{ccc}
0 & 1 & 0 \\
0 & 0 & 1 \\
-a_{0} & -a_{1} & -a_{2}
\end{array}\right] \text {, } \\
& B_{F}=Q_{O} B=\left[\begin{array}{c}
0 \\
0 \\
\mathbf{b} \frac{S_{1} S_{2} S_{3}}{P_{1} P_{2} P_{3}}
\end{array}\right]=\left[\begin{array}{c}
0 \\
0 \\
b_{0}
\end{array}\right] \text {, } \\
& C_{F}=C Q_{O}^{-1}=\left[\begin{array}{lll}
1 & 0 & 0
\end{array}\right] \text {. }
\end{aligned}
$$

By differentiating $y(t)$ twice in (10), we get (13).

It is clear that the following statements are true:

- if a step change (fault) occurs in the flow rate coefficient $S_{1}$, then step changes will be seen in $z_{1}(t)$ and in the next state variables $z_{2}(t)$ and $z_{3}(t)$;

- if a step change occurs in the flow rate coefficient $S_{2}$, then step changes will be seen only in the state variables $z_{2}(t)$ and $z_{3}(t)$;

- if a step change occurs in the flow rate coefficient $S_{3}$, then a step change will be seen only in the state variable $z_{3}(t)$.

The above statements can be generalized to any number of cascade-connected tanks.

Below, we will analyze another system with the structure of series-connected tanks.

\section{Second example system with faults}

As the second example of (1), we shall consider a three-tank series-connected system as in Fig. 4, given by

$$
\begin{aligned}
{\left[\begin{array}{l}
\dot{x}_{1}(t) \\
\dot{x}_{2}(t) \\
\dot{x}_{3}(t)
\end{array}\right]=} & {\left[\begin{array}{ccc}
\frac{-S_{1}}{P_{1}}-\frac{S_{2}}{P_{1}} & \frac{S_{2}}{P_{1}} & 0 \\
\frac{S_{2}}{P_{2}} & -\frac{S_{2}}{P_{2}}-\frac{S_{3}}{P_{2}} & \frac{S_{3}}{P_{2}} \\
0 & \frac{S_{3}}{P_{3}} & -\frac{S_{3}}{P_{3}}
\end{array}\right] } \\
& \times\left[\begin{array}{l}
x_{1}(t) \\
x_{2}(t) \\
x_{3}(t)
\end{array}\right]+\left[\begin{array}{c}
0 \\
0 \\
\frac{\mathbf{b}}{P_{3}}
\end{array}\right] u(t),
\end{aligned}
$$

$$
\begin{gathered}
y(t)=\left[\begin{array}{lll}
S_{1} & 0 & 0
\end{array}\right] x(t), \\
G(s)=\frac{b_{0}}{s^{3}+a_{2} s^{2}+a_{1} s+a_{0}}, \\
b_{0}=\frac{\mathbf{b} S_{1} S_{2} S_{3}}{P_{1} P_{2} P_{3}}, \\
a_{2}=\frac{S_{1}}{P_{1}}+\frac{S_{2}}{P_{2}} \frac{P_{1}+P_{2}}{P_{1}} \\
+\frac{S_{3}}{P_{3}} \frac{P_{2}+P_{3}}{P_{2}}, \\
a_{1}=\frac{S_{1} S_{2}}{P_{1} P_{2}}+\frac{S_{1} S_{3}}{P_{1} P_{3}} \frac{P_{2}+P_{3}}{P_{2}} \\
+\frac{S_{2} S_{3}}{P_{2} P_{3}} \frac{P_{1}+P_{2}+P_{3}}{P_{1}}, \\
a_{0}=\frac{S_{1} S_{2} S_{3}}{P_{1} P_{2} P_{3}} .
\end{gathered}
$$

The observability matrix $Q_{O}[3 \times 3]$ and matrices $F, B_{F}$, $C_{F}$ are

$$
\begin{aligned}
& Q_{O}=\left[\begin{array}{c}
C \\
C A \\
C A^{2}
\end{array}\right] \\
& =S_{1}\left[\begin{array}{ccc}
1 & 0 & 0 \\
-\frac{S_{1}+S_{2}}{P_{1}} & \ldots & 0 \\
\frac{\left(S_{1}+S_{2}\right)^{2}}{P_{1}^{2}}+\frac{S_{2}^{2}}{P_{1} P_{2}} & \cdots & \cdots
\end{array}\right] \\
& =\left[\begin{array}{cc}
\cdots & 0 \\
\cdots & \frac{S_{2}}{P_{1}} \\
\cdots & -\frac{S_{2}\left(S_{1}+S_{2}\right)}{P_{1}^{2}}-S_{2} \frac{S_{2}+S_{3}}{P_{1} P_{2}}
\end{array}\right.
\end{aligned}
$$

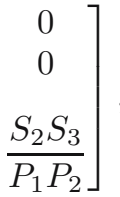

$$
\begin{aligned}
& F=Q_{O} A Q_{O}^{-1}=\left[\begin{array}{ccc}
0 & 1 & 0 \\
0 & 0 & 1 \\
-a_{0} & -a_{1} & -a_{2}
\end{array}\right] \text {, } \\
& B_{F}=Q_{O} B=\left[\begin{array}{c}
0 \\
0 \\
\frac{\mathbf{b} S_{1} S_{2} S_{3}}{P_{1} P_{2} P_{3}}
\end{array}\right]=\left[\begin{array}{c}
0 \\
0 \\
b_{0}
\end{array}\right] \text {, } \\
& C_{F}=C Q_{O}^{-1}=\left[\begin{array}{lll}
1 & 0 & 0
\end{array}\right] \text {. }
\end{aligned}
$$

By differentiating $y(t)$ twice in (14), we obtain (17). It is easy to see that once more the following statements are true: 


$$
\begin{aligned}
& {\left[\begin{array}{l}
z_{1}(t) \\
z_{2}(t) \\
z_{3}(t)
\end{array}\right]=\left[\begin{array}{l}
y(t) \\
\dot{y}(t) \\
\ddot{y}(t)
\end{array}\right]} \\
& =S_{1}\left[\begin{array}{ccc}
1 & 0 & 0 \\
-\frac{S_{1}}{P_{1}} & \frac{S_{2}}{P_{1}} & 0 \\
\frac{S_{1}^{2}}{P_{1}^{2}} & -\frac{S_{1} S_{2}}{P_{1}^{2}}-\frac{S_{2}^{2}}{P_{1} P_{2}} & \frac{S_{2} S_{3}}{P_{1} P_{2}}
\end{array}\right]\left[\begin{array}{l}
x_{1}(t) \\
x_{2}(t) \\
x_{3}(t)
\end{array}\right] \\
& =\left[\begin{array}{c}
S_{1} x_{1}(t) \\
-\left(\frac{S_{1}}{P_{1}}\right) S_{1} x_{1}(t)+\left(\frac{S_{1}}{P_{1}}\right) S_{2} x_{2}(t) \\
\left(\frac{S_{1}}{P_{1}}\right)^{2} S_{1} x_{1}(t)-\left[\left(\frac{S_{1}}{P_{1}}\right)^{2}+\frac{S_{1} S_{2}}{P_{1} P_{2}}\right] S_{2} x_{2}(t)+\frac{S_{1} S_{2}}{P_{1} P_{2}} S_{3} x_{3}(t)
\end{array}\right] .
\end{aligned}
$$

$$
\begin{aligned}
{\left[\begin{array}{l}
z_{1}(t) \\
z_{2}(t) \\
z_{3}(t)
\end{array}\right]=} & {\left[\begin{array}{l}
y(t) \\
\dot{y}(t) \\
\ddot{y}(t)
\end{array}\right] } \\
= & S_{1}\left[\begin{array}{ccc}
1 & 0 & 0 \\
-\frac{S_{1}+S_{2}}{P_{1}} & \cdots & 0 \\
\frac{\left(S_{1}+S_{2}\right)^{2}}{P_{1}^{2}}+\frac{S_{2}^{2}}{P_{1} P_{2}}, & \cdots & \cdots
\end{array}\right]\left[\begin{array}{l}
x_{1}(t) \\
x_{2}(t) \\
x_{3}(t)
\end{array}\right] \\
= & S_{1}\left[\begin{array}{crr}
1 & 0 \\
\cdots & \frac{S_{2}}{P_{1}} & \\
\cdots & -\frac{S_{2}\left(S_{1}+S_{2}\right)}{P_{1}^{2}}-\frac{S_{2}\left(S_{2}+S_{3}\right)}{P_{1} P_{2}} & \frac{S_{2} S_{3}}{P_{1} P_{2}}
\end{array}\right]\left[\begin{array}{l}
x_{1}(t) \\
x_{2}(t) \\
x_{3}(t)
\end{array}\right]
\end{aligned}
$$

- if a step change (fault) occurs in the flow rate coefficient $S_{1}$, then step changes will be seen in $z_{1}(t)$ and in the next state variables $z_{2}(t)$ and $z_{3}(t)$;

- if a step change occurs in the flow rate coefficient $S_{2}$, then step changes will only be seen in the state variables $z_{2}(t)$ and $z_{3}(t)$;

- if a step change occurs in the flow rate coefficient $S_{3}$, then a step change will only be seen in the state variable $z_{3}(t)$.

The above statements can be generalized to any number of series-connected tanks. For fault localization it is enough to carefully observe all the $n-1$ derivatives of the output signal $y(t)$, i.e., all canonical state variables $z(t)$ of the system of series-connected tanks as well as cascade, connected ones. To this end, we shall use the idea of the double window exact state observer.

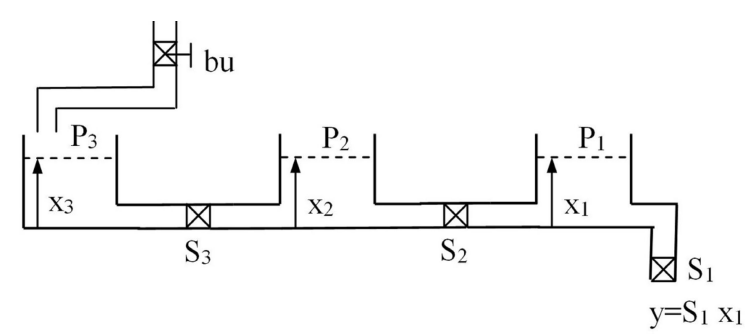

Fig. 4. Three tank serial connected system.

\section{Simulation experiments for the first system}

The results of application of the above described diagnostic methodology for the third order system (10) of cascade-connected tanks with the control of $u(t)=\mathbf{b} 1(t)$, $\mathbf{b}=10$, will be presented in two experiments. 

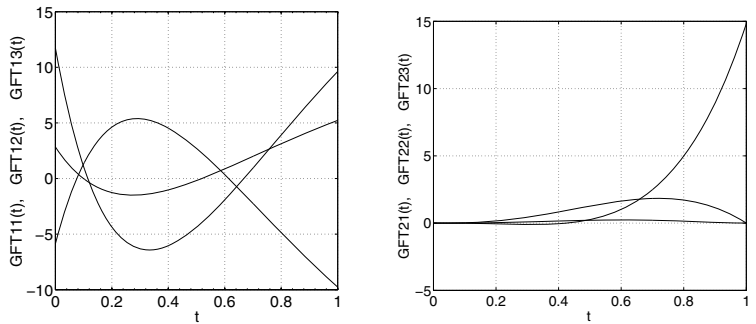

Fig. 5. Functions $G_{F T 1}(t), G_{F T 2}(t)$ for the left window.

8.1. First experiment for the first system. The cross-sectional area of tanks is $P_{1}=1.5, P_{2}=4.5, P_{3}=$ 1. The coefficients are $S_{1}=5, S_{2}=2, S_{3}=1$. The step change (fault) occurs in the flow rate of coefficient $S_{1}$. Before fault occurrence, the value of coefficient $S_{1}$ was $S_{1}=5$, and after the fault it is $S_{1}=8$. The interval of experiment is $[0,4]$. The moment of fault occurrence is $t_{A}=2$. The width of the observation windows is $T=1$. Before the fault the Frobenius matrix is $F$, and afterwards it is $\bar{F}$, cf. (12). We have

$$
\begin{aligned}
F & =\left[\begin{array}{ccc}
0 & 1 & 0 \\
0 & 0 & 1 \\
-1.4815 & -5.2593 & -4.7778
\end{array}\right], \\
\bar{F} & =\left[\begin{array}{ccc}
0 & 1 & 0 \\
0 & 0 & 1 \\
-2.3704 & -8.1481 & -6.778
\end{array}\right], \\
B_{F}= & {\left[\begin{array}{lll}
0 & 0 & 14.8148
\end{array}\right]^{T}, } \\
\bar{B}_{F}= & {\left[\begin{array}{lll}
0 & 0 & 23.7037
\end{array}\right]^{T}, } \\
M_{F O}^{-1}= & {\left[\begin{array}{ccc}
15 & -105 & 492 \\
-105 & 953 & -4780 \\
492 & -4780 & 24577
\end{array}\right], } \\
\bar{M}_{F O}^{-1}= & {\left[\begin{array}{ccc}
18 & -162 & 1025 \\
-162 & 1755 & -11740 \\
1025 & -11740 & 79412
\end{array}\right] . }
\end{aligned}
$$

The function matrices

$$
\begin{aligned}
& G_{F T 1}(t)=\left[\begin{array}{l}
G_{F T 1_{1}}(t) \\
G_{F T 1_{2}}(t) \\
G_{F T 1_{3}}(t)
\end{array}\right], \\
& G_{F T 2}(t)=\left[\begin{array}{l}
G_{F T 2_{1}}(t) \\
G_{F T 2_{2}}(t) \\
G_{F T 2_{3}}(t)
\end{array}\right]
\end{aligned}
$$

for reconstruction of the final state in the left-side window (before the fault) calculated from (9) in the window $[0,1]$ have the form of two function vectors (for the SISO system) whose elements are functions presented in Fig. 5. The function matrices $\bar{G}_{F 01}(\tau)$ and $\bar{G}_{F 02}(\tau)$ for the initial state in the right-side window (after the fault) calculated from (6) have the form as in Fig. 6.
The simulation results of reconstruction of the derivatives of the output $y$ (3) generated by the double window observer (8) (left window) and (5) (right window) are shown below. Figures 7-9 confirm the statement from Section 6 about the step changes in $z(t)$.
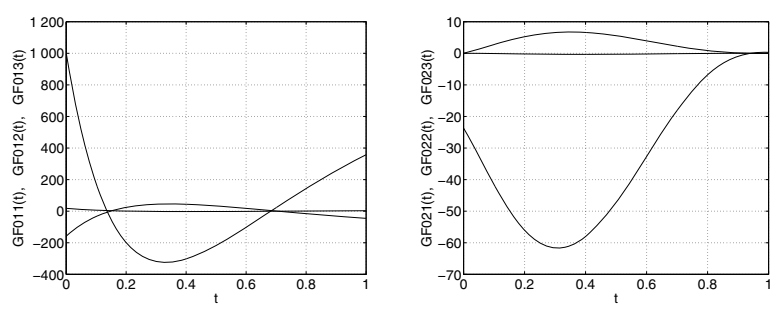

Fig. 6. Functions $\bar{G}_{F T 1}(\tau), \bar{G}_{F T 2}(\tau)$ for the left window.

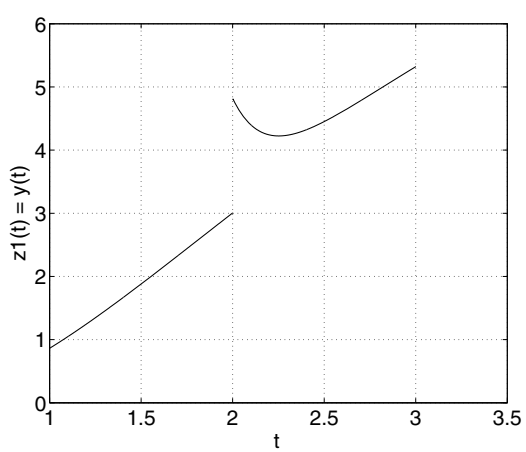

Fig. 7. Step change in the output $y(t)=z_{1}(t)$.

There is visible reconstruction of the final state $z_{T}(t)$ by the left moving window $[t-1, t]$ before the fault for $t \in[1,2]$, as well as reconstruction of the initial state $z_{0}(t)$ by the right moving window $[t-1, t]$ after the fault for $t \in$ $[3,4]$ (the last reconstructed initial state value is $z_{0}(3)$ ). In all state variables $z_{i}(t)$, step changes at $t=2$ occur, meaning that the fault happened in parameter $S_{1}$.

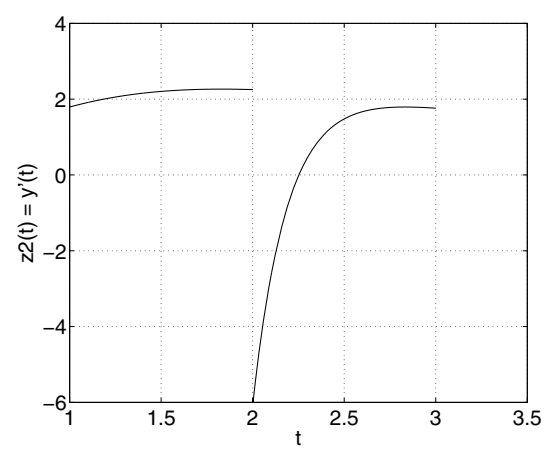

Fig. 8. First derivative of the output $\dot{y}(t)=z_{2}(t)$. 


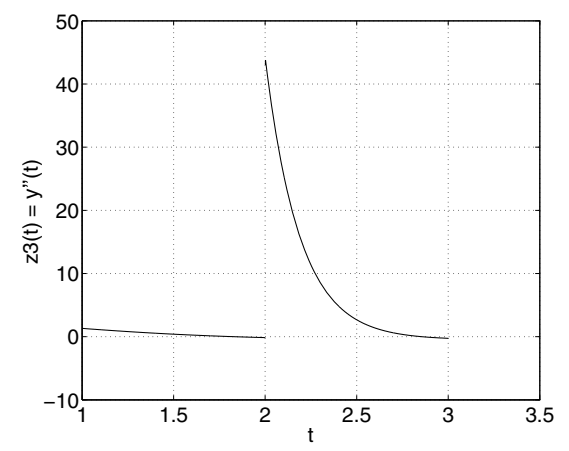

Fig. 9. Second derivative of the output $\ddot{y}(t)=z_{3}(t)$.
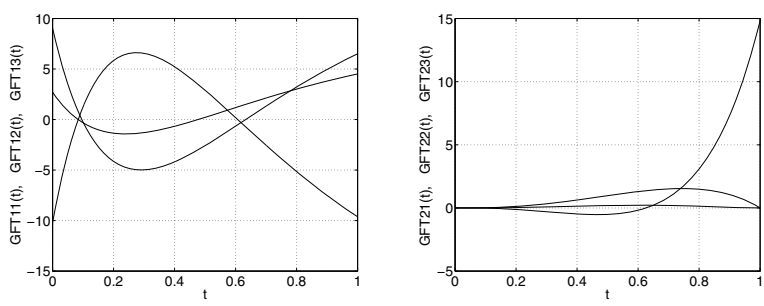

Fig. 10. Matrices $G_{F T 1}(t)$ and $G_{F T 2}(t)$ for the left window.

8.2. Second experiment for the first system. The cross-sectional area of the tanks is $P_{1}=1.5, P_{2}=4.5$, $P_{3}=1$. The coefficients are $S_{1}=2, S_{2}=1, S_{3}=5$. A step change (fault) occurs in the flow rate coefficient $S_{3}$, before the fault $S_{3}=5$ and after the fault $S_{3}=8$. The interval of experiment is $[0,4]$. The moment of fault occurrence is $t_{A}=2$. The width of the observation window is $T=1$. Before fault occurrence, the Frobenius matrix is marked as $F$, and after the fault as $\bar{F}$, cf. (12):

$$
\begin{aligned}
F & =\left[\begin{array}{ccc}
0 & 1 & 0 \\
0 & 0 & 1 \\
-1.4815 & -8.0741 & -6.5556
\end{array}\right], \\
\bar{F} & =\left[\begin{array}{ccc}
0 & 1 & 0 \\
0 & 0 & 1 \\
-2.3704 & -12.7407 & -9.5555
\end{array}\right], \\
B_{F} & =\left[\begin{array}{lll}
0 & 0 & 14.8148
\end{array}\right]^{T}, \\
\bar{B}_{F} & =\left[\begin{array}{lll}
0 & 0 & 23.7037
\end{array}\right]^{T}, \\
M_{F O}^{-1} & =\left[\begin{array}{ccc}
18 & -152 & 924 \\
-152 & 1627 & -10498 \\
924 & -10498 & 60956
\end{array}\right], \\
\bar{M}_{F O}^{-1} & =\left[\begin{array}{ccc}
20 & -260 & 2280 \\
-260 & 3550 & -32350 \\
2280 & -32350 & 297390
\end{array}\right] .
\end{aligned}
$$

The function matrices $G_{F T 1}(t)$ and $G_{F T 2}(t)$ and for the final state in the left-side window (before the fault) calculated from (8) have the form as in Fig. 10. The
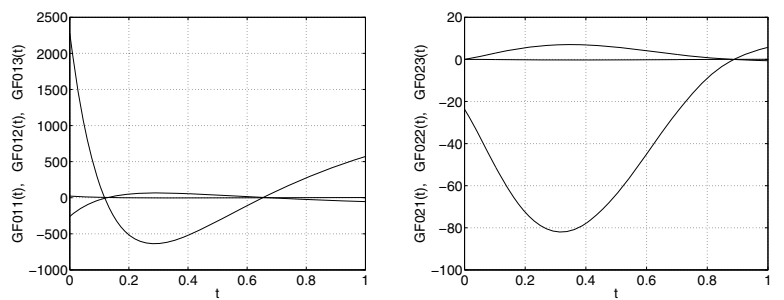

Fig. 11. Matrices $\bar{G}_{F 01}(\tau)$ and $\bar{G}_{F 02}(\tau)$ for the left window.

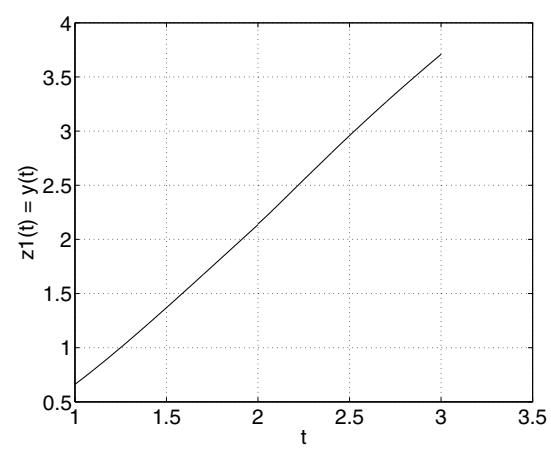

Fig. 12. Output $y(t)=z_{1}(t)$ without step changes.

function matrices $\bar{G}_{F 01}(\tau)$ and $\bar{G}_{F 02}(\tau)$ for the initial state in the right-side window (after the fault) calculated from (6) have the form as in Fig. 11. The simulation results of reconstruction of the derivatives of the output $y$ (3) generated by the double window observer based on (16) (left window) and (14) (right window) are shown in Figs. 12-14. In these figures one can find once more a confirmation of the statement from Section 6 about step changes in $\left.z_{(} t\right)$. In this experiment the failure occurred in the parameter $S_{3}$, hence the step changes should be visible only on the $z_{3}(t)$ variable of the state (the second derivative of $y(t)$ ). The figures present the reconstructed derivatives in interval $\left[t_{A}-T, t_{A}+T\right]$ as in Fig. 2.

\section{Simulation experiments for the second system}

In this section the results of application of the above described diagnostic methodology will be presented in two experiments for series-connected tanks (system (14)) with the control of $u(t)=\mathbf{b} 1(t), \mathbf{b}=10$ (see Fig. 4).

9.1. First experiment for the second system. The cross-sectional area of the tanks is $P_{1}=1.5, P_{2}=4.5$ and $P_{3}=1$. The coefficients are $S_{1}=2, S_{2}=1$, $S_{3}=5$. The step change (fault) occurs in the flow rate of coefficient $S_{1}$. Before fault occurrence, the value of coefficient $S_{1}$ was $S_{1}=5$, and after the fault it is $S_{1}=8$. The interval of experiment is $[0,4]$. The 


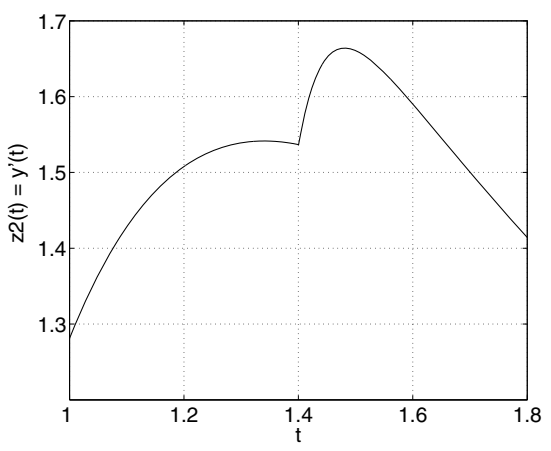

Fig. 13. First derivative of the output $\dot{y}(t)=z_{2}(t)$.

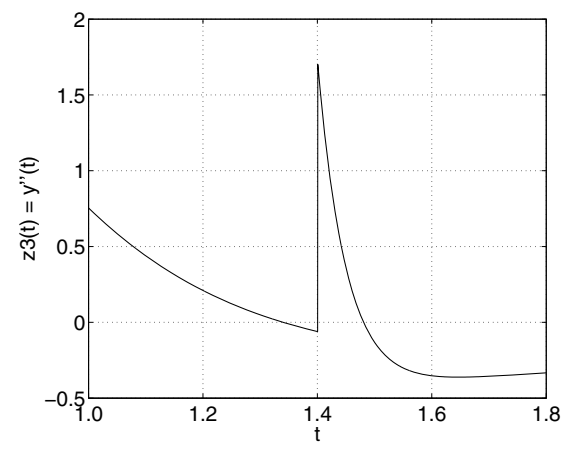

Fig. 14. Second derivative of the output $\ddot{y}(t)=z_{3}(t)$.

moment of fault occurrence is $t_{A}=2$. The width of the observation window is $T=0.5$. Before fault occurrence, the Frobenius matrix is $F$ and after the fault it is $\bar{F}$, cf. (16):

$$
\begin{aligned}
F & =\left[\begin{array}{ccc}
0 & 1 & 0 \\
0 & 0 & 1 \\
-1.4815 & --7.6296 & -6.3333
\end{array}\right], \\
\bar{F} & =\left[\begin{array}{ccc}
0 & 1 & 0 \\
0 & 0 & 1 \\
-2.3704 & -10.963 & -8.333
\end{array}\right] \\
B_{F} & =\left[\begin{array}{lll}
0 & 0 & 14.8148
\end{array}\right]^{T}, \\
\bar{B}_{F} & =\left[\begin{array}{ccc}
0 & 0 & 23.7037
\end{array}\right]^{T}, \\
M_{F O}^{-1} & =\left[\begin{array}{ccc}
30 & -300 & 2240 \\
-300 & 953 & -37230 \\
2240 & -37230 & 303440
\end{array}\right], \\
\bar{M}_{F O}^{-1} & =\left[\begin{array}{ccc}
30 & -380 & 3400 \\
-380 & 6570 & -62250 \\
3400 & -62250 & 603090
\end{array}\right]
\end{aligned}
$$
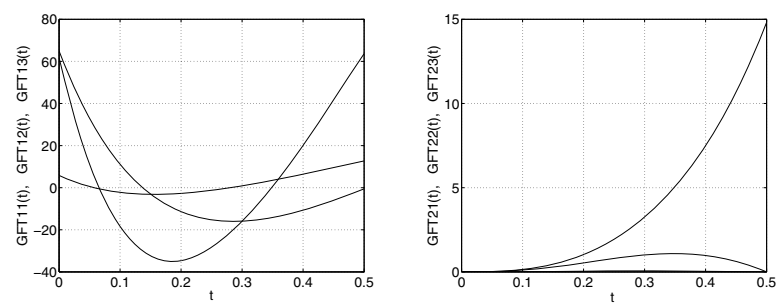

Fig. 15. Matrices $G_{F T 1}(t)$ and $G_{F T 2}(t)$ for the left window.
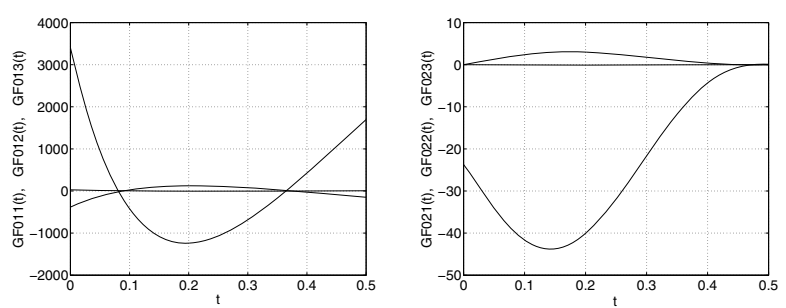

Fig. 16. Matrices $\bar{G}_{F 01}(\tau)$ and $\bar{G}_{F 02}(\tau)$ for the right window.

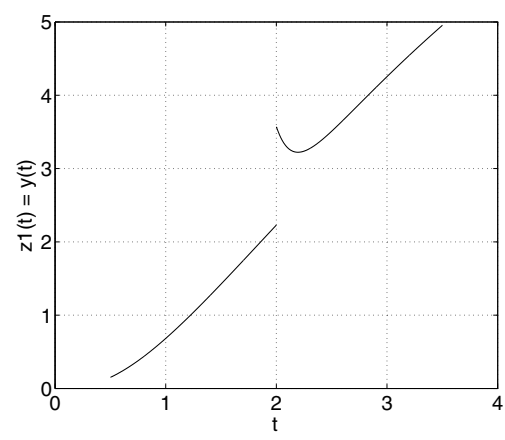

Fig. 17. Step change in the output $y(t)=z_{1}(t)$.

The function matrices

$$
\begin{aligned}
& G_{F T 1}(t)=\left[\begin{array}{l}
G_{F T 1_{1}}(t) \\
G_{F T 1_{2}}(t) \\
G_{F T 1_{3}}(t)
\end{array}\right], \\
& G_{F T 2}(t)=\left[\begin{array}{l}
G_{F T 2_{1}}(t) \\
G_{F T 2_{2}}(t) \\
G_{F T 2_{3}}(t)
\end{array}\right]
\end{aligned}
$$

for the final state of the left-side window (before the fault) calculated from (8) have the form as in Fig. 15. The function matrices $\bar{G}_{F 01}(\tau)$ and $\bar{G}_{F 02}(\tau)$ for the initial state of the right-side window (after fault occurrence) calculated from (5) have the form as in Fig. 16. The simulated reconstruction results of the derivatives of the output $y$ (3) generated by the double window observer (8) (left window) and (5) (right window) are shown below. In Figs. 17-19, one can find a confirmation of the statement from Section 7 about the step changes in $z(t)$. There is visible reconstruction of the final state $z_{T}(t)$ by the left-side moving window $[t-0.5, t]$ before the fault for 


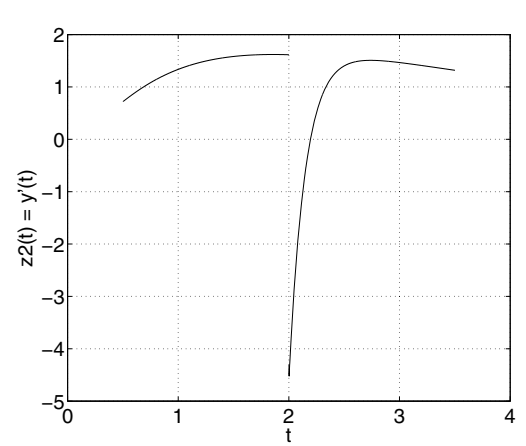

Fig. 18. First derivative of the output $\dot{y}(t)=z_{2}(t)$.

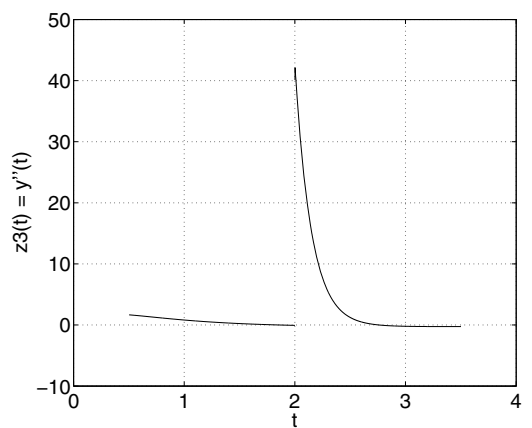

Fig. 19. Second derivative of the output $\ddot{y}(t)=z_{3}(t)$.

$t \in[0.5,2]$, as well as reconstruction of the initial state $z_{0}(t)$ by the right-side moving window $[t-0.5, t]$ after fault occurrence for $t \in[2.5,4]$. The step changes occur in all state variables $z_{i}(t)$, meanings that the fault happened in parameter $S_{1}$.

9.2. Second experiment for the second system. The cross-sectional area of the tanks is $P_{1}=1.5, P_{2}=4.5$ and $P_{3}=1$. The coefficients are $S_{1}=2, S_{2}=1, S_{3}=5$. The step change (fault) occurs it the flow rate coefficient $S_{3}$. Before the fault the value of $S_{3}$ was $S_{3}=5$, and after fault occurrence it is $S_{3}=8$. The interval of experiment is $[0,4]$. The moment of fault is $t_{A}=2$. The width of the observation window is $T=0.5$. Before the fault the Frobenius matrix is denoted by $F$ and after the fault by $\bar{F}$, cf. (16):

$$
\begin{aligned}
F & =\left[\begin{array}{ccc}
0 & 1 & 0 \\
0 & 0 & 1 \\
-1.4815 & -13.6296 & -8.3333
\end{array}\right], \\
\bar{F} & =\left[\begin{array}{ccc}
0 & 1 & 0 \\
0 & 0 & 1 \\
-2.3704 & -21.6296 & -12.0
\end{array}\right], \\
B_{F} & =\left[\begin{array}{lll}
0 & 0 & 14.8148
\end{array}\right]^{T}, \\
\bar{B}_{F} & =\left[\begin{array}{lll}
0 & 0 & 23.7037
\end{array}\right]^{T},
\end{aligned}
$$

$$
\begin{aligned}
M_{F O}^{-1}= & {\left[\begin{array}{ccc}
30 & -380 & 3280 \\
-380 & 6470 & -60260 \\
3280 & -60260 & 575630
\end{array}\right], } \\
\bar{M}_{F O}^{-1}= & {\left[\begin{array}{ccc}
0 & -600 & 6500 \\
-600 & 11400 & -141200 \\
6500 & -141200 & 1769700
\end{array}\right], }
\end{aligned}
$$

The function matrices $G_{F T 1}(t)$ and $G_{F T 2}(t)$ for the final state of the left-side window (before the fault) calculated from (8) have the form as in Fig. 20. The function matrices $\bar{G}_{F 01}(\tau)$ and $\bar{G}_{F 02}(\tau)$ for the initial state of the right-side window (after the fault) calculated from (5) have the form as in Fig. 21. The simulation results of reconstructing
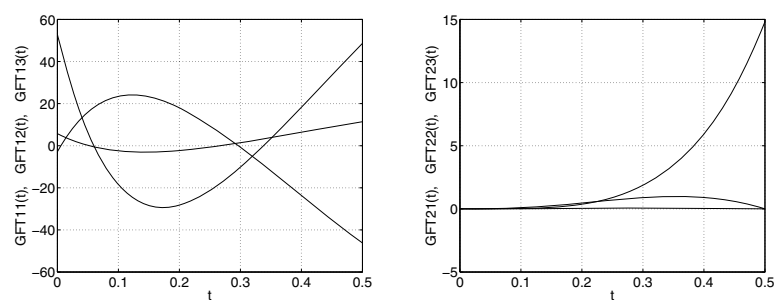

Fig. 20. Matrices $G_{F T 1}(t)$ and $G_{F T 2}(t)$ for the left window.
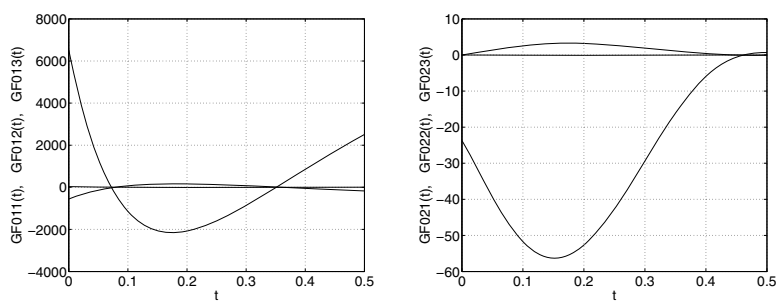

Fig. 21. Matrices $\bar{G}_{F 01}(\tau)$ and $\bar{G}_{F 02}(\tau)$ for the right window.

the derivatives of the output $y$ (3) generated by the double window observer based on (8) (left-side window) and (5) (right-window) are shown in Figs. 22, 23 and 24. There one can find a confirmation of the statement from Section 7 about the step changes in $z(t)$. In the figures, reconstruction of the final state $z_{T}(t)$ by the left moving window in $[t-0.5, t]$ before the moment of fault is visible, for $t \in[0.5,2]$, along with the reconstruction of the initial state $z_{0}(t)$ by the right moving window in $[t-0.5, t]$ after the fault for $t \in[2.5,4]$.

One can see that the step change at $t_{A}=2$ occurs only in the state $z_{3}(t)$, meaning that the failure happened on parameter $S_{3}$.

\section{Diagnosis of a more general system}

Let us discuss diagnosis of abrupt changes of parameters in more general structures of SISO linear systems. The transfer function of the input-output model will have the 
form

$$
G(s)=\frac{b_{n-1} s^{n-1}+\cdots+b_{1} s+b_{0}}{s^{n}+\cdots+a_{2} s^{2}+a_{1} s+a_{0}} .
$$

Assume that there is a possibility of identification of all the model parameters $a_{i}, b_{i}$ before and immediately after the moment of fault occurrence. The question is whether the exact calculation of step changes in real parameters of the system (symbolically denoted by the letter $S_{i}$ ) based on these model parameters values is possible. Various cases were analyzed by Byrski (2014), and a short description of these cases will be presented below. As an example of analysis, we assume once more a third-order hydraulic system in the configuration as in Fig. 25, for which the input-output model is

$$
G(s)=\frac{b_{2} s^{2}+b_{1} s+b_{0}}{s^{3}+a_{2} s^{2}+a_{1} s+a_{0}} .
$$

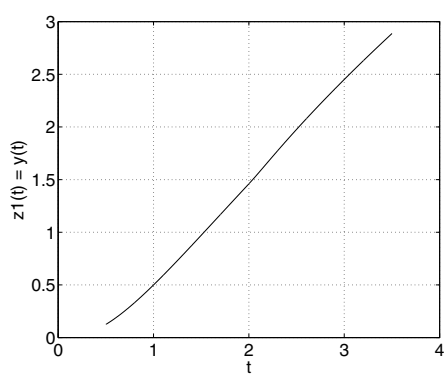

Fig. 22. Reconstructed output $y(t)=z_{1}(t)$.

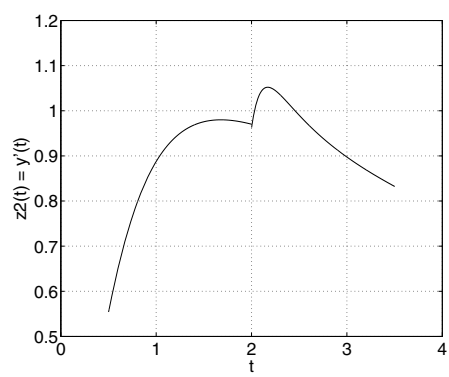

Fig. 23. Reconstructed first derivative $\dot{y}(t)=z_{2}(t)$.

For simplicity of the description, we will assume the equality of the tanks cross-sectional area to be $P_{1}=P_{2}=$ $P_{3}=P=1$. Then the real state model of the system is given by the equations

$$
\begin{aligned}
{\left[\begin{array}{l}
\dot{x_{1}}(t) \\
\dot{x_{2}}(t) \\
\dot{x_{3}}(t)
\end{array}\right] } & =\left[\begin{array}{ccc}
-S_{1} & S_{2} & 0 \\
0 & -S_{2} & S_{3} \\
0 & 0 & -S_{3}
\end{array}\right]\left[\begin{array}{l}
x_{1}(t) \\
x_{2}(t) \\
x_{3}(t)
\end{array}\right]+\left[\begin{array}{l}
\mathbf{b}_{1} \\
\mathbf{b}_{2} \\
\mathbf{b}_{3}
\end{array}\right] u(t), \\
y(t) & =\left[\begin{array}{lll}
S_{1} & 0 & 0
\end{array}\right] x(t) .
\end{aligned}
$$

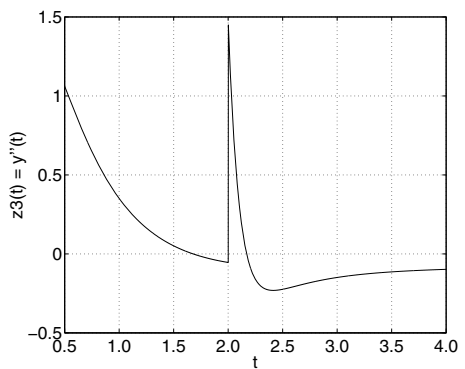

Fig. 24. Second derivative of the output $\ddot{y}(t)=z_{3}(t)$.

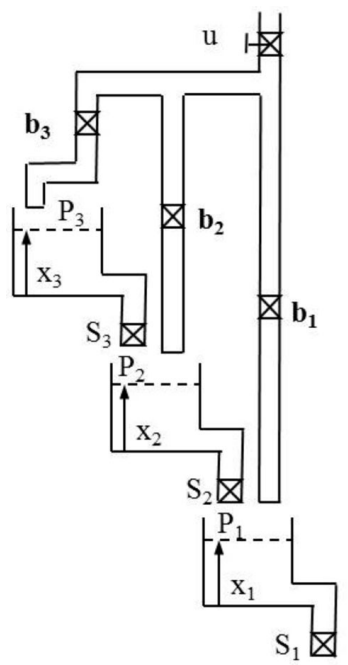

Fig. 25. Three tank cascade system.

One can obtain the transfer function (19) from the formula $G(s)=C[s I-A]^{-1} B$. Then the model parameters are given by the formulas

$$
\begin{aligned}
& b_{2}=\mathbf{b}_{1} S_{1} \\
& b_{1}=\mathbf{b}_{1} S_{1} S_{2}+\mathbf{b}_{1} S_{1} S_{3}+\mathbf{b}_{2} S_{1} S_{2}, \\
& b_{0}=\left(\mathbf{b}_{1}+\mathbf{b}_{2}+\mathbf{b}_{3}\right) S_{1} S_{2} S_{3}, \\
& a_{2}=S_{1}+S_{2}+S_{3} \\
& a_{1}=S_{1} S_{2}+S_{1} S_{3}+S_{2} S_{3}, \\
& a_{0}=S_{1} S_{2} S_{3} .
\end{aligned}
$$

Case 1. If the model parameters $a_{i}, b_{i}$ are identified before and after the failure and the gain coefficients $\mathbf{b}_{\mathbf{1}}, \mathbf{b}_{\mathbf{2}}, \mathbf{b}_{\mathbf{3}}$ are known, then from the above equations one can find that

$$
\begin{aligned}
& S_{1}=\frac{b_{2}}{\mathbf{b}_{1}} \\
& S_{3}=\frac{b_{2}\left(\mathbf{b}_{1}+\mathbf{b}_{2}\right)\left(\mathbf{b}_{1} a_{2}-b_{2}\right)-\mathbf{b}_{1}^{2} b_{1}}{\mathbf{b}_{1} b_{2}\left(\mathbf{b}_{1}+\mathbf{b}_{2}\right)-\mathbf{b}_{1}^{2} b_{2}} \\
& S_{2}=\frac{a_{0}}{S_{1} S_{3}} .
\end{aligned}
$$


This means that before the moment of fault occurrence as well as immediately after it, by a calculation of the above equations the old and new values of system parameters $S_{i}$ are known directly. Hence, the faulty parameter $S_{F}$ is easy to recognize and there is no need to use the diagnostic method presented in this paper.

Case 2. Another situation occurs if the gain coefficient $\mathbf{b}_{1}=0$. The transfer function has the form

$$
G(s)=\frac{b_{1} s+b_{0}}{s^{3}+a_{2} s^{2}+a_{1} s+a_{0}},
$$

and the model parameters are given by

$$
\begin{aligned}
& b_{1}=\mathbf{b}_{2} S_{1} S_{2}, \\
& b_{0}=\left(\mathbf{b}_{2}+\mathbf{b}_{3}\right) S_{1} S_{2} S_{3}, \\
& a_{2}=S_{1}+S_{2}+S_{3}, \\
& a_{1}=S_{1} S_{2}+S_{1} S_{3}+S_{2} S_{3}, \\
& a_{0}=S_{1} S_{2} S_{3} .
\end{aligned}
$$

After some calculation one can obtain the quadratic equation for $S_{1}$. There are two possible solutions of this equation:

$$
\begin{array}{rlrl}
\Delta & =\left(a_{2}+a_{0} \frac{\mathbf{b}_{2}}{b_{1}}\right)^{2}-4 \frac{b_{1}}{\mathbf{b}_{2}}, & \\
S_{11,12} & =\frac{1}{2}\left(a_{2}+a_{0} \frac{\mathbf{b}_{2}}{b_{1}} \pm \sqrt{\Delta}\right), & \\
S_{21} & =\frac{b_{1}}{\mathbf{b}_{2}} \frac{1}{S_{11}}, & S_{31} & =\frac{a_{0}}{S_{11} S_{21}}, \\
S_{22} & =\frac{b_{1}}{\mathbf{b}_{2}} \frac{1}{S_{12}}, & S_{32} & =\frac{a_{0}}{S_{12} S_{22}} .
\end{array}
$$

In this case, after identification of new values of the parameters $a_{i}, b_{i}$ (after faults) and calculation of the possible values of parameters $S_{i}$, direct recognition of the faulty parameter $S_{F}$ may be possible, but in some special malicious cases it may be not.

Case 3. Yet another case of possible situations is for $\mathbf{b}_{1}=$ $\mathbf{b}_{2}=0$ and $\mathbf{b}_{3}=\mathbf{b} \neq 0$. The state equation is given by Eqns. (10) and (11):

$$
G(s)=\frac{b_{0}}{s^{3}+a_{2} s^{2}+a_{1} s+a_{0}} .
$$

From (11), after some algebra one can obtain the formula for $S_{1}$. This is a third-degree equation which has three possible solutions for $S_{11}, S_{12}, S_{13}$. For each $S_{1 j}$ there is a second-degree equation for $S_{2}$, hence two possible solutions for $S_{2 j}$ exist and finally one solution for $S_{3 j}$ :

$-S_{1}^{3}+a_{2} S_{1}^{2}-a_{1} S_{1}+a_{0}=0$,

$\stackrel{3 S_{1 j}}{\Longrightarrow} S_{2}^{2}-\left(a_{2}-S_{1 j}\right) S_{2}+\frac{a_{0}}{S_{1 j}}=0$,

$\Longrightarrow S_{2 j}=\frac{1}{2}\left(a_{2}-S_{1 j}+\sqrt{\left(a_{2}-S_{1 j}\right)^{2}-4 \frac{a_{0}}{S_{1 j}}}\right)$,

$S_{2 j}=\frac{1}{2}\left(a_{2}-S_{1 j}-\sqrt{\left(a_{2}-S_{1 j}\right)^{2}-4 \frac{a_{0}}{S_{1 j}}}\right)$

$S_{3 j}=\frac{a_{0}}{S_{1 j} S_{2 j}}$

In this case there are six possible sets of the same triple values $S_{i}$, but arranged in a different sequence. Direct, correct and fast diagnosis as well as location of the fault in this case may be impossible. The standard approach is to carry out numerical experiments and testing the different hypotheses regarding the different sequences of the parameter values $S_{i}$ with simulation of the abrupt changes in consecutive $S_{i}$ 's. After this, by comparing the real output signal and different modeling output signals, a meaningful hypothesis must be chosen that will enable determination of the correct $i$-th number of the faulty valve. This methodology is impossible for on-line applications.

It is clear that the third case with $\mathbf{b}_{1}=\mathbf{b}_{2}=0$ and $\mathbf{b}_{3}=\mathbf{b} \neq 0$ is most complicated for diagnosis. Hence, especially for this case (and also for the second one), the method presented in this paper is intended. This method does not use information about the changed value of $S_{i}$; however, it can indicate the position of the faulty valve in the system structure.

\section{Identification method}

Although the method of model identification which cooperates with the double window observer is not the main subject of this paper, a brief description of this method will be presented here along with comments on this cooperation. In the literature (e.g., Young, 1981; Unbehauen and Rao, 1987), various methods of parameter identification of time-continuous models have been discussed. For the diagnostic algorithm presented in this paper the identification method with a modulating function is most useful. The efficiency of this method has been investigated by the authors and the results of the identification published (Byrski and Byrski, 2012b). The improvement of this method which enables acceleration of calculations was developed and the results were published by Byrski and Byrski (2012a).

The above method for identification of parameters of the linear continuous $n$-th order input-output model

$$
\sum_{i=0}^{n} a_{i} y^{(i)}(t)=\sum_{j=0}^{m} b_{j} u^{(j)}(t)
$$


is based on convolution transformation of both the sides of this model with the special known modulating function $\varphi(t)$ with compact support $[0, h]$; that is to say, $\varphi(t)$ and $\varphi^{(i)}(t)$ are given on the interval $(0, h)$, and are zero outside this interval (Preisig and Rippin, 1993), $h<T_{I D}$ :

$$
\begin{gathered}
\sum_{i=0}^{n} a_{i}\left[\int_{0}^{h} y(t-\tau) \varphi^{(i)}(\tau) \mathrm{d} \tau\right] \\
=\sum_{j=0}^{m} b_{j}\left[\int_{0}^{h} u(t-\tau) \varphi^{(j)}(\tau) \mathrm{d} \tau\right], \\
\sum_{i=0}^{n} a_{i} y_{i}(t)=\sum_{j=0}^{m} b_{j} u_{j}(t) .
\end{gathered}
$$

After such transformation of the differential model, a new algebraic model is obtained which contains the new functions $y_{i}(t), u_{j}(t)$ that replace the unknown derivatives $y^{(i)}(t), u^{(i)}(t)$. This algebraic model has the same parameters (however, still unknown) and is valid for any initial conditions. Parameter identification may be performed based on the equation error method. The use of minimization of the integral square error in the moving integral window $\left[t-T_{I D}, t\right]$ for any $t$ generates a special Gram matrix. The eigenvector of this matrix represents the best estimate of the system parameters $a_{i}(t), b_{i}(t)$. The identification moving window cooperates with the left-side window of the double window observer $[t-2 T, t-T]$ (where $T>T_{I D}$ ) and produces, for the observer, the current values of the input-output model parameters $a_{i}, b_{i}$ before the fault (for calculation of matrices $G_{F T 1}(t), G_{F T 2}(t)$ ), as well as cooperates with the right-side window of the double window observer $[t-$ $T, t]$ and yields, for this observer, the current values of the input-output model parameters $\bar{a}_{i}(t), \bar{b}_{i}(t)$ after the fault (for calculation of new matrices $\bar{G}_{F 01}(\tau), \bar{G}_{F 02}(\tau)$ ). In an on-line application, all calculations of the identification procedure in the entire identification window $T_{I D}$ must be completed and repeated within each single measurement sample.

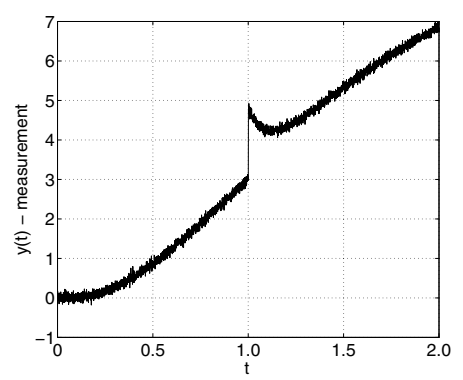

Fig. 26. Noisy measurement of the output $y(t)$.

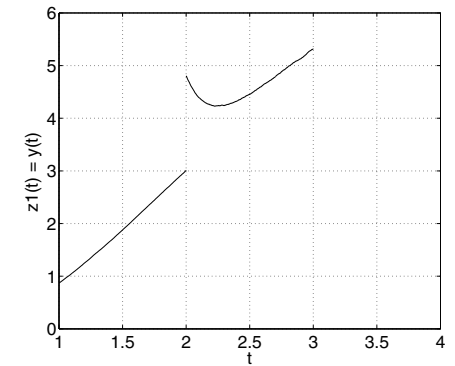

Fig. 27. Reconstructed output signal $y(t)=z_{1}(t)$.

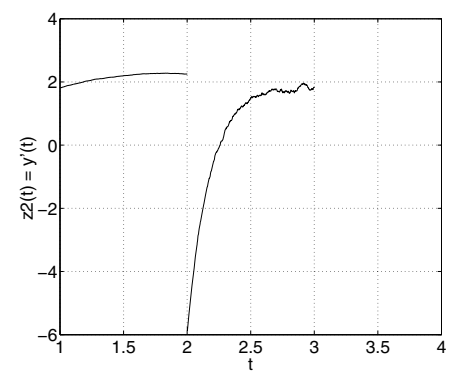

Fig. 28. Reconstructed first derivative $\dot{y}(t)=z_{2}(t)$.

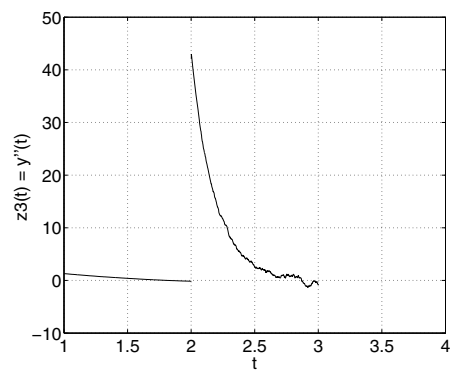

Fig. 29. Second derivative of output $\ddot{y}(t)=z_{3}(t)$.

\section{Conclusions}

The main and original contribution of this paper is the new method for diagnosis of a system and localization of some real parameter $S_{i}$ in which an abrupt change has occurred, by observation of the canonical state of this system. This method only requires knowledge of the input-output model with identified values of the model parameters $a_{i}, b_{i}$ and does not require knowledge of the real parameter values $S_{i}$. The step changes in the observed canonical state variables indicate the $i$-th number of the real parameter $S_{i}$ which has failed. The correctness of this methodology has been shown for linear systems with a regular structure (cascade or serial connections of first order systems). Such a structure can be found in many real systems, e.g., in series or cascade connections of irrigation reservoirs or technological tanks, and in electric cable modeling with RC elements in chain or in series connections of manipulator arms. Many authors have carried out research on such structures (e.g., Theilliol 
et al., 2002; Sainz et al., 2002; Lincon et al., 2007; Orani et al., 2010; Wang et al., 2008).

Another contribution of this paper is the new idea of observer construction. It proposes the use of the double moving window integral observer for observation of the exact value of the canonical state. The integral observer plays the role of a high quality virtual sensor for reconstruction of the output signal derivative. This observer reproduces derivatives $y^{(i)}(t)$ very efficiently and has good filtration properties because of the two integral operations (for signal processing). It works properly even if a noisy signal $y(t)$ is measured. It is visible in extra simulation results made for the first system in the first experiment presented in Section 8 (Figs. 7-9), but this time with noisy measurements $y(t)$. The results can be seen in Figs. 26-29.

Due of considering the continuous time problem, the observation interval $T$ can be theoretically of any length, so it can be chosen to be as short as possible. In practice, for guaranteeing the accuracy of numerical calculation of the integrals and from the point of view of possible existence of disturbances in input/output measurements, this interval should have a reasonable duration and could be chosen by an experiment. For the integral observer, one can calculate its norm as the functional norm in $L^{2}[0, T]$. This norm depends on the system matrices $A, B, C$ as well as the observation interval $T$. If in the measurement signals the disturbances occur and they have some norm in $L^{2}[0, T]$, then the norm of the observation error can be estimated (as an upper estimate) by the product of the norm of disturbances and the norm of the observer.

An important remark is that, if the observation interval $T$ increases, the norm of the observer decreases. On the other hand, if the interval $T$ decreases, then the norm of the observer increases and so does the influence of the disturbance to the observation error.

The exact state observers (6), (9) chosen in this paper are optimal from the point of view of disturbances, which are present only in measurements of the output signal $y(t)$. We assume that the control signal is known exactly. This means that in both observers only the first integral must have the minimal norm. Then the influence of the additive disturbances of the output measurements will be minimized (Byrski, 2003).

The methods of fault detection which uses finite memory integral observers have been rarely studied and reported. One can find only few publications in which such an approach is presented (e.g., Medvedev, 1996; Nuninger et al., 1998). Commonly for diagnostic purposes, the asymptotic Luenberger estimator is applied. However, because of the initial state observation error, as has already been stated, this estimator can only estimate the state and cannot reconstruct this state exactly in a finite time interval. The presented diagnostic algorithm was implemented in a software package and it has been embedded as a separate task to the STERGLASS computer control system. Such a system was established at the AGH University of Science and Technology in Kraków and is dedicated to the monitoring and control of industrial glass melting installations.

\section{Acknowledgment}

This work was partially supported with the funds for the grant PBS2/A3/16/2013 (National Center for Research and Development in Poland).

\section{References}

Basseville, M. and Nikiforov, I.V. (1993). Detection of Abrupt Changes. Theory and Application, Prentice Hall, Englewood Cliffs, NJ.

Blanke, M., Kinnaert, M., Lunze, J. and Staroswiecki, M. (2003). Diagnosis and Fault-Tolerant Control, Springer, Berlin.

Byrski, J. (2014). Finite Memory Algorithms for Signal Processing in the Diagnosis of Processes, Ph.D., thesis, AGH University of Science and Technology, Kraków.

Byrski, J. and Byrski, W. (2012a). Design and implementation of a new algorithm for fast diagnosis of step changes in parameters of continuous systems, 8th IFAC Symposium on Fault Detection Supervision and Safety for Technical Processes, SAFEPROCESS'12, Mexico City, Mexico, pp. 695-700.

Byrski, W. (1995). Theory and application of the optimal integral state observers, 3rd European Control Conference, ECC'95, Rome, Italy, pp. 52-66.

Byrski, W. (2003). The survey for the exact and optimal state observers in Hilbert spaces, 7th European Control Conference, ECCO3, Cambridge, UK.

Byrski, W. and Byrski, J. (2012b). The role of parameter constraints in EE and $\mathrm{OE}$ methods for optimal identification of continuous LTI models, International Journal of Applied Mathematics and Computer Science 22(2): 379-388, DOI: 10.2478/v10006-012-0028-3.

Byrski, W. and Fuksa, S. (1996). Linear adaptive controller for continuous system with convolution filter, Proceedings of the IFAC 13th Triennial World Congress, San Francisco, CA, USA, pp. 379-384.

Carlsson, B., Ahlen, A. and Sternad, M. (1991). Optimal differentiation based on stochastic signal models, IEEE Transactions on Signal Processing 39(2): 341-353.

Chen, J. and Patton, R. (1999). Robust Model-Based Fault Diagnosis for Dynamic Systems, Kluwer Academic, Boston, MA.

Chen, J. and Zhang, H. (1991). Robust detection of faulty actuators via input observers, International Journal of System Science 22(10): 1829-1839.

Chiang, L., Russell, E. and Braatz, R. (2001). Fault Detection and Diagnosis in Industrial Systems, Springer, London. 
Costa, O.L., Fragoso, M.D. and Marques, R.P. (2005). DiscreteTime Markov Jump Linear Systems, Springer, Berlin.

Costa, O.L., Fragoso, M.G. and Todorov, M.G. (2013). Continuous-Time Markov Jump Linear Systems, Springer, Berlin.

Ding, X. (2013). Model-Based Fault Diagnosis Techniques, Springer, London.

Ding, X. and Guo, L. (1996). Observer-based fault detection, 13th IFAC World Congress, San Francisco, CA, USA, pp. 157-162.

Frank, P.M. (1990). Fault diagnosis in dynamic systems using analytical and knowledge-based redundancy-a survey and some new results, Automatica 26(3): 459-474.

Fuksa, S. and Byrski, W. (1984). General approach to linear optimal estimator of finite number of parameters, IEEE Transactions on Automatic Control 29(5): 470-472.

Ibir, S. (2004). Linear time-derivative trackers, Automatica 40(3): 397-405.

Isermann, R. (2006). Fault-Diagnosis Systems, Springer, Berlin.

Jouffroy, J. and Reger, J. (2015). Finite-time simultaneous parameter and state estimation using modulating functions, IEEE Conference on Control Applications (CCA), Sydney, Australia, pp. 394-399.

Korbicz, J., Kościelny, J.M., Kowalczuk, Z. and Cholewa, Z. (Eds.) (2004). Fault Diagnosis. Models, Artificial Intelligence, Application, Springer, Berlin.

Lai, T.L. and Shan, J.Z. (1999). Efficient recursive algorithms for detection of abrupt changes in signals and control systems, IEEE Transactions on Automatic Control 44(5): 952-966.

Lincon, S.A., Sivakumar, D. and Prakash, J. (2007). State and fault parameter estimation applied to three-tank bench mark relying on augmented state Kalman filter, ICGST Journal of Automatic Control and System Engineering 7(1): 33-41.

Medvedev, A. (1996). Fault detection and isolation by functional continuous deadbeat observers, International Journal of Control 64(3): 425-439.

Niedzwiecki, M. (1994). Identification of time-varying systems with abrupt parameter changes, Automatica 30(3): 447-459.

Nuninger, W., Kratz, F. and Ragot, J. (1998). Finite memory generalised state observer for failure detection in dynamic systems, IEEE Conference on Decision \& Control, Tampa, FL, USA, pp. 581-585.

Orani, N., Pisano, A. and Usai, E. (2010). Fault diagnosis for the vertical three-tank system via high-order sliding-mode observation, Journal of the Franklin Institute 347(6): 923-939.
Patton, E., Frank, P. and Clark, R. (2000). Issues of Fault Diagnosis for Dynamic Systems, Springer, Berlin.

Preisig, H.A. and Rippin, D.W.T. (1993). Theory and application of the modulating function method, Computers and Chemical Engineering 17(1): 1-16.

Qu, R. (1996). A new approach to numerical differentiation and integration, Mathematical and Computer Modelling 24(10): 55-68.

Reger, J. and Jouffroy, J. (2009). On algebraic time-derivative estimation and deadbeat state reconstruction, IEEE Conference on Decision and Control, Shanghai, China, pp. 1740-1745.

Rolink, M., Boukhobza, T. and Sauter, D. (2006). High order sliding mode observer for fault actuator estimation and its application to the three tanks benchmark, German-French Institute for Automation and Robotics,

http: //hal.archives-ouvertes.fr/ hal-00121029/en/.

Sainz, M., Armengol, J. and Vehi, J. (2002). Fault detection and isolation of the three-tank system using the modal interval analysis, Journal of Process Control 12(2): 325-338.

Simani, S., Fantuzzi, C. and Patton, R. (2003). Model Based Fault Diagnosis in Dynamic Systems Using Identification Techniques, Springer, London.

Smith, M.S., Moes, T.R. and Morelli, E.A. (2003). Real-time stability and control derivative extraction from F-15 flight data, AIAA Atmospheric Flight Mechanics Conference and Exhibit, Austin, TX, USA, p. 5701.

Theilliol, D., Noura, H. and Ponsart, J.C. (2002). Fault diagnosis and accommodation of a three-tank system based on analytical redundancy, ISA Transactions 41(3): 365-382.

Ukil, A. and Zivanovic, R. (2007). Application of abrupt change detection in power systems disturbance analysis and relay performance monitoring, IEEE Transactions on Power Delivery 22(1): 365-382.

Unbehauen, H. and Rao, G.P. (1987). Identification of Continuous Systems, North Holland, Amsterdam.

Vainio, O., Renfors, M. and Saramaki, T. (1997). Recursive implementation of FIR differentiators with optimum noise attenuation, IEEE Transactions on Instrumentation and Measurement 46(5): 1202-1207.

Wang, W., Bo, Y., Zhou, K. and Ren, Z. (2008). Fault detection and isolation for nonlinear systems with full state information, 17th IFAC World Congress, Seoul, Korea, pp. 901-909.

Wei, T., Hon, Y.C. and Wang, Y.B. (2005). Reconstruction of numerical derivatives from scattered noisy data, Inverse Problems 21(2): 657-672.

Young, P. (1981). Parameter estimation for continuous-time models-a survey, Automatica 17(1): 23-39. 


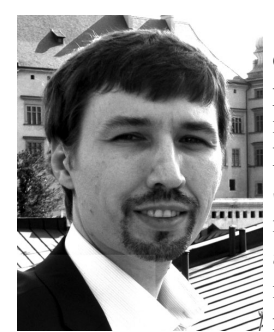

Jedrzej Byrski obtained the Ph.D. degree in computer science in 2014. Presently he is employed as an assistant professor at the Department of Applied Computer Science, Faculty of Electrical Engineering, Automatics, Computer Science and Biomedical Engineering, AGH-UST in Cracow. His fields of research cover methods and algorithms for safety problems in computer networks, firewall software, the CORBA system, real time computer systems under the QNX operating environment, as well as mathematical algorithms for identification and fault detection in computer control systems. He has participated in many scientific research projects within domestic grants (Polish Committee of Scientific Research and the Ministry of Science and Higher Education) as well as European research projects (University of Birmingham, UK), designing the interface for CLP-Prolog software package for operational control of water systems). He is also involved in teaching as a specialist in computer graphics.

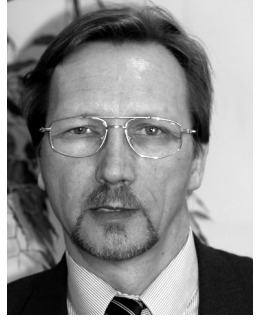

Witold Byrski obtained the Ph.D. degree in automatic control in 1976 from the AGH University of Science and Technology in Cracow, Poland. He received his doctor habilitatus (D.Sc.) degree in 1994, and in 2009 was granted the professorial title. Presently he is a full professor in the Department of Automatics and Biomedical Engineering DABE (Process Control Group), Faculty of Electrical Engineering, Automatics, Computer Science and Biomedical Engineering, AGH-UST in Cracow. Since 1998 he has been the deputy head of the department. His fields of research cover problems of computer control systems and, in particular, integral algorithms for exact state observation, as well as convolution methods for identification of continuous dynamic models. The results of his research have been published in a monograph, a textbook, papers and international conferences or congresses (over 100 reviewed publications and over 100 other research reports).

Received: 18 June 2015

Revised: 26 November 2015

Re-revised: 20 March 2016

Accepted: 26 April 2016 